\title{
Editorial
}

\section{Tricotilomanía, tricofagia y el Síndrome de Rapunzel}

La tricotilomanía (arrancarse su propio pelo) fue descrita en la bibliografía por el psiquiatra francés Hallopeau, en 1889, y fue aceptada como un diagnóstico psiquiátrico casi cien años después, en 1987. Su incidencia se considera que oscila entre el $0.6 \%$ y el $1.6 \%$. De las personas con tricotilomanía solo el $30 \%$ se come el pelo (tricofagia) y de ese porcentaje se estima que un $1 \%$ requerirá tratamiento quirúrgico para remover el tricobezoar.

Cuando el tricobezoar tiene una cola que se extiende hasta pasar el duodeno, se da una sintomatología diferente conocida con el nombre del síndrome de Rapunzel.

Se le llamó así, ya que "poéticamente” esta problemática evocó el cuento de los hermanos Wilhelm y Jacob Grimm, escrito en 1812, que habla de una joven de cabello tan largo que tiraba sus trenzas para que el principe subiera. A los padres de Rapunzel, por comer la fruta prohibida del jardín de la bruja, esta les quitó su hija y la encerró en una torre. Pero eventualmente el príncipe que la rescata, logra salvarla al subir por medio de sus trenzas.

Los pacientes tricofagia son presa de su adicción comer pelos y no es sino hasta que "un síntoma” asoma, que el médico o cirujano acusioso descubre y “salva a la joven princesa”.

El síndrome de Rapunzel tiene su origen en la tricotilomanía, que unida a una tricofagia produce a lo largo del tiempo un tricobezoar. O sea que un problema psiquiátrico es la causa de una "cirugía". La bibliografía señala que en un $90 \%$ de los casos los tricobezoares se reportan en mujeres, y en menores de 30 años, el $80 \%$. El diagnóstico se suele complicar porque el paciente con frecuencia oculta su tricofagia.

Los pacientes se arrancan el pelo de la cabeza, las cejas, las pestañas, brazos, piernas y pubis. No en todas las personas que se "arrancan" el pelo se produce alopecia. En un porcentaje de aproximadamente un 1.5\% de los hombres se vuelve crónico, y en un 3.4\% de las mujeres así sucede.

Dos tipos de tricotilomanía se han descrito en función del desorden, y pueden existir, solos o a la vez. El "focalizado" consiste en que la persona se detiene en tiempo y atención para halar el pelo. Este tipo se ha asociado a una sensación de tensión previa a halar el pelo, que mejora una vez que este se arranca. El "automático o sedentario" se caracteriza porque la persona se hala el pelo cuando descansa, ve televisión y no se percata de que lo hace, hasta que descubren el pelo en su mano.

Entre un $40 \%$ y un 70\% de las personas que se halan el pelo hacen algo más con el juegan, se comen la raíz, lo pasan por los labios o se lo comen. Cuando la tricotilomanía aparece antes de los 10 años es más de tipo automático o sedentario y suele tener mejor pronóstico. Si la tricotilomanía ocurre en la adolescencia, se describe que suele evolucionar a la cronicidad y acompañarse de otros trastornos psiquiátricos; es más frecuente en mujeres que en varones.

Algunos autores consideran la tricotilomanía como parte de "las conductas repetitivas centradas en el cuerpo" (Bohne, et al. 2002), es decir todos aquellos comportamientos de una persona, en los cuales se arranca partes de su cuerpo: como pelo, uñas o mucosas, y que son parte de lo que se conoce como un trastorno del control de impulsos. 
Las complicaciones de la tricotilomanía, en general, son rash o eritema del área, alopecia, infecciones del cuero cabelludo y hasta síndrome del túnel carpal. Cuando las personas se comen el pelo, el riesgo es que se forme un tricobezoar y se produzca un problema de absorción intestinal, con todas las complicaciones propias de los bezoar.

En cuanto al tratamineto, los niños y niñas requieren una evaluación por psiquiatría de niños y adolescentes, para aclarar diagnósticos y establecer los respectivos tratamientos con el fin de evitar una recaída o cronicidad del caso, dentro de lo posible.

Se ha descrito en la bibliografía que el uso de inhibidores de recaptura de serotonina, por ser una conducta repetitiva (compulsiva), ha mostrado mejores resultados que el placebo. Eso sí, podría llegar a requerir dosis altas, de fluoxetina hasta $60 \mathrm{mgs}$, o de sertralina $100 \mathrm{mgs}$, como se utiliza para los trastornos obsesivos, pues investigaciones parecen indicar que se produce por una disfunción serotoninérgica.

Sin embargo, algunos estudios no concuerdan con que el uso de medicamentos sea el mejor tratamiento de la tricotilomanía y recomiendan la terapia conductual. La experiencia clínica se inclinar más por un modelo mixto de terapia conductual y medicación.

Yasmín Maria Jaramillo-Borges

Psiquiatra General y Especialista en Niños

y Adolescentes

MSc. Gerencia Salud

Jefe del Servicio de Psiquiatría del HNN

Miembro Correspondiente Academia de Medicina.

\section{Referencias}

1. Aguilar A, Rodríguez G, Hernández C. Síndrome de Rapunzel: reporte de un caso. Acta Méd Costarric. [online]. jun. 2003, vol.45, no.2 [citado 01 Agosto 2006], p.80-83.

2. Frei, A, et al: Hari apparente: Rapunzel syndrome., AJP. 162:2, Febrero 2005

3. Velasco S, Paredes E. Tricobezoar: un problema psicológico, . . An Esp Pediatr 2001; 55: 383 - 384

4. Balik E, Ulman I, Taneli C, Demircan M. The Rapunzel syndrome: A case report and review of the literature. Eur J Pediatr Surg 1993; 3: 171-173.

5. Bohne A, Wilhelm S, Keuthen NJ, Baer L, Jenike MA. Skin picking in German students: Prevalence, phenomenology, and associated characteristics. Behav Modif. 2002; 26: 320 - 33

6. Ocampo del Prado, LC, Cervantes R, Mata N., Zárate F, Ramírez-J. Tricobezoar. Informe de un caso interesante. Presentación de un caso y revisión de literatura. Acta Pediatr Méx 2003;24:194-7

7. Sharma V, Sharma ID. Intestinal trichobezoar with perforation in a child. J Pediatr Surg 1992; 27: 518-519.

8. Deslypere JP, Praet M, Verdonk G: An unusual Case of the Trichobezoar: The Rapunzel Syndrome. Am J Gastroenterol 1982; 77: 467-70.

9. Ponce J, Gomollón F, de Argila de Prados C., Mínguez M, Miño Fugarolas G.” Tratamiento de las enfermedades gastroenterológicas” Asociación Gastroenterológica Española. Asociación Española de Gastroenterología, Secretaría Técnica: Ediciones Doyma, S.L. Travessera de Gracia, 17 - 2º 08021 Barcelona.

10. Van Minnen, A, et al, Treatment of Trichotillomania With Behavioral Therapy or Fluoxetine A Randomized, Waiting-List Controlled Study. Arch Gen Psychiatry. 2003;60:517-522.

11. Juan Carlos Martínez Aguayo *, Claudia González Larenas *, Eduardo Correa Donoso "Conductas repetitivas centradas en el cuerpo", páginas 40-44, Revista APAL,

12. Palmer J, Yates R, Trotter Lisa, Childhood Trichotillomania, Successful Treatment With Fluoxetine Following an SSRI Failure Psychosomatics 40:526-528, December 1999The Academy of Psychosomatic Medine. 